\section{Safety and efficacy of intravitreal injection of recombinant erythropoietin for protection of photoreceptor cells in a rat model of retinal detachment}

\begin{abstract}
Purpose To elucidate the safety and efficacy of exogenous erythropoietin (EPO) for the protection of photoreceptor cells in a rat model of retinal detachment (RD).

Methods Recombinant rat EPO (400 ng) was injected into the vitreous cavity of normal rats to observe the eye manifestations. Retinal function was assessed by flash electroretinograms. Histopathological examination of retinal tissue was performed at $\mathbf{1 4}$ days and $\mathbf{2}$ months after injection, respectively. To investigate the inhibitory effect of EPO on photoreceptor cell apoptosis in RD rats, 100, 200, or $400 \mathrm{ng}$ EPO was injected into the vitreous cavity immediately after RD model establishment. Apoptosis of photoreceptor cells was determined at 3 days after injection. Caspase- 3 activation was measured by western blot analysis and immunofluorescence, respectively, and the level of Bcl- $X_{L}$ expression was analyzed by western blot.
\end{abstract}

Results Intravitreal injection of EPO $400 \mathrm{ng}$ into normal rats had no significant impact on retinal function, morphology, or structure. Apoptosis of retinal photoreceptor cells apparently increased after RD and was significantly reduced following EPO treatment. The thickness of the outer nuclear layer in the RD $+400 \mathrm{ng}$ group was significantly thicker than that in other experimental RD groups both at 14 days and at 2 months after RD $(P<0.05)$. Western blot and immunofluorescence analyses showed
Z Xie ${ }^{1,2,3,4}$, F Chen ${ }^{1,2,4}, X$ Wu $^{3}$, C Zhuang ${ }^{1,2}$, J Zhu ${ }^{1,2}$, J Wang $^{1,2}, \mathrm{H} \mathrm{Ji}^{1,2}, \mathrm{Y}$ Wang $^{1,2}$ and $X \mathrm{Hua}^{1,2}$ decreased caspase- 3 activation and increased Bcl- $\mathrm{X}_{\mathrm{L}}$ expression following EPO treatment. Conclusion Intravitreal injection of EPO $400 \mathrm{ng}$ is safe, and EPO may suppress caspase-3 activation and enhance $\mathrm{Bcl}-\mathrm{X}_{\mathrm{L}}$ expression, resulting in inhibition of apoptosis and protection of photoreceptor cells. Eye (2012) 26, 144-152; doi:10.1038/eye.2011.254; published online 21 October 2011

Keywords: erythropoietin; retinal detachment/ experimental; flash electroretinogram; apoptosis

Introduction

Erythropoietin (EPO) is an erythropoiesisstimulating factor secreted by the embryonic liver and adult kidney under hypoxic conditions. It has an anti-apoptotic effect, and promotes the proliferation and differentiation of erythroid progenitor cells. ${ }^{1}$ An increasing number of studies have demonstrated the neuroprotective effects of EPO and its receptor, by anti-apoptotic mechanisms. Several studies have indicated that EPO can protect photoreceptor cells from the effects of lightinduced and hereditary retinal dystrophy; ${ }^{2-5}$ protect retinal neurons from ischemiareperfusion injury, ${ }^{6}$ and retinal ganglion cells after acute and chronic ocular hypertension; ${ }^{7-9}$ promote ganglion cell survival and axonal regeneration after optic nerve transection; ${ }^{10-12}$ attenuate inflammation in multiple sclerosis optic neuritis; ${ }^{13,14}$ reduce the permeability of the retinal barrier and protect retinal neurons in 
diabetic retinopathy; ${ }^{15-18}$ relieve diabetic macular edema; ${ }^{19}$ enhance the stability of hypoxic retinal vessels; ${ }^{20,21}$ and has a neuroprotective role in pigment epithelial cells subjected to photo-oxidative damage. ${ }^{22}$

EPO exerts its biological effects through interaction with EPO receptor (EPOR). Studies have confirmed EPO and EPOR expression in human embryonic and adult retinas, ${ }^{23-25}$ and in the normal rat retina. ${ }^{10,11}$ EPOR is expressed in the inner segment of photoreceptor cells, ganglion cells, the presynaptic outer plexiform layer, and in the inner and outer nuclear layers (ONL). ${ }^{10,11}$

Retinal detachment (RD) is a common blindnesscausing eye disorder, characterized by separation of the neural retina from the retinal pigment epithelium. The separation of the neural retina prevents substance exchange with the retinal pigment epithelium, and results in loss of nutrient supply from the choroid. The main secondary pathological changes include the collapse and shortening of the photoreceptor outer segment, as well as photoreceptor cell apoptosis, ${ }^{26}$ thus seriously affecting functional recovery after RD. Our previous study determined that the expression levels of EPO and EPOR were upregulated after RD, reaching a peak at $48 \mathrm{~h} .{ }^{27}$ Clinical studies have also demonstrated elevated levels of intravitreal EPO after RD. ${ }^{28}$ Our previous studies confirmed that EPO in the detached retinas was neutralized by EPOR antagonists, thereby aggravating photoreceptor cell apoptosis after RD (in press). These lines of evidence indicate that the endogenous EPO/EPOR system may protect retinal photoreceptor cells after RD, and suggest that exogenous EPO supplementation could also help to reduce RD-induced damage to retinal photoreceptor cells. However, the safety and efficacy of supplementary EPO remains unclear. This study aimed to elucidate the safety, efficacy, and molecular mechanisms of intravitreal injection of EPO in normal and RD rats, in terms of its protective effect on retinal photoreceptor cells.

\section{Materials and methods}

\section{Experimental animals}

Normal male SD rats, aged 10 weeks, weighing $250 \pm 20 \mathrm{~g}$, were purchased from the Shanghai Experimental Animal Center of the Chinese Academy of Sciences, China. All experimental protocols were performed in strict accordance with the guidelines for the Use of Animals in Ophthalmic and Visual Research issued by the Association for Research in Vision and Ophthalmology. All rats were fed and maintained under a 12-h light/dark cycle (0800-2000 light and 2000-0800 dark) at $22-25^{\circ} \mathrm{C}$ and $55-60 \%$ humidity.

\section{RD models}

As previously described, ${ }^{27}$ rats were anesthetized by injecting Sumianxin (Institute of Military Veterinarian, Academy of Military Medical Sciences, Jilin, China) into the thigh muscle $(0.6 \mathrm{ml} / \mathrm{kg})$, followed by local administration of three drops of tropicamide to induce mydriasis, and oxybuprocaine for corneal surface anesthesia. Under an operating microscope (Leica, Bensheim, Germany), a sclerotomy was created approximately $2 \mathrm{~mm}$ posterior to the limbus with a 25-gauge pinhead, with special caution taken to avoid damaging the lens. $1.4 \%$ sodium hyaluronate (Healon GV; Pharmacia \& Upjohn, Uppsala, Sweden) was slowly injected into the peripheral subretinal space in the superior neurosensory retina, thus detaching it from the underlying retinal pigment epithelium. In each experimental eye, approximately one half of the retina was detached in the superior.

\section{Intravitreal administration of EPO}

Rats were anesthetized using the above methods and subjected to mydriasis. Under the operating microscope (Leica), a sclerotomy was created approximately $2 \mathrm{~mm}$ posterior to the limbus with a 25-gauge pinhead, taking special caution to avoid damaging the lens. A total of $5 \mu \mathrm{l}$ of phosphate-buffered saline (PBS) or $400 \mathrm{ng}$ of EPO (R\&D Systems, Minneapolis, MN, USA) was administered using a microinjector along the puncture incision. Ofloxacin eye ointment was applied after the surgery. Once the RD model was successfully established, the vitreous cavity was immediately injected with a single injection of $5 \mu \mathrm{l}$ PBS, or 100,200 , or $400 \mathrm{ng}$ EPO respectively.

\section{Flash electroretinogram (ERG) recording}

Rats were anesthetized by injecting $0.6 \mathrm{ml} / \mathrm{kg}$ Sumianxin into the thigh muscle, followed by oxybuprocaine for corneal surface anesthesia and tropicamide for mydriasis. After 30-min dark adaptation, the maximal retinal response and oscillatory potentials were recorded using a Tomey-EP1000 (Tomey, Tokyo, Japan). The recording parameters were as follows: stimulator, Ganzfeld Q400; intensity of stimulation light, $3 \mathrm{~cd} \mathrm{~s} / \mathrm{m}^{2}$; stimulation frequency, $0.11 \mathrm{~Hz}$; cycle time, $9.091 \mathrm{~ms}$; sampling frequency, $2560 \mathrm{~Hz}$ for $0.39 \mathrm{~ms}$; band pass, 0.2 $300 \mathrm{~Hz}$. Ops: stimulation frequency, $0.07 \mathrm{~Hz}$; cycle time, $14.286 \mathrm{~ms}$; sampling frequency, $5120 \mathrm{~Hz}$ for $0.2 \mathrm{~ms}$; band pass, $100-500 \mathrm{~Hz}$; stacking three times. The recording electrode with a circular metal electrode was located on the limbus coated with ofloxacin eye ointment, and the reference electrode was placed subcutaneously in the 
frontal region. Earth electrodes were placed subcutaneously on the back.

\section{Terminal deoxynucleotidyl transferase dUTP nick end labeling (TUNEL) in situ examination of apoptosis}

As previously described, ${ }^{29}$ conventional dewaxing was performed according to the instructions of the kit. Samples were incubated with proteinase $\mathrm{K}$, and then treated with a dropwise TUNEL reaction mixture (Promega, Madison, WI, USA). The nuclei were finally stained with propidium iodide (PI, Sigma, St Louis, MO, USA). Samples were observed under a laser scanning confocal microscope (Zeiss 510, Zeiss, Jena, Germany). Apoptotic photoreceptor cells in the retina were stained with yellow fluorescence.

\section{Western blot analysis}

Retinas were resuspended in the lysis buffer $(30 \mathrm{mM}$ Tris, $\mathrm{pH} 7.5,150 \mathrm{mM} \mathrm{NaCl}, 1 \mathrm{mM}$ PMSF, $1 \mathrm{mM} \mathrm{Na} \mathrm{VO}_{4}, 1 \%$ Nonidet P-40, and $10 \%$ glycerol) and then centrifuged for $10 \mathrm{~min}$ at $4{ }^{\circ} \mathrm{C}$. Protein concentration of the supernatant (protein fraction) was calculated using the BCA protein assay. An aliquot of $40 \mu \mathrm{g}$ total extracts was mixed with protein loading buffer containing 2-ME and boiled for 5 min before loading onto an SDS/10\% polyacrylamide gel. After electrophoresis, proteins were transferred onto nitrocellulose membranes. Nonspecific binding was blocked with 5\% non-fat dry milk in Tween/ Tris-buffered saline, and then membranes were incubated overnight at $4{ }^{\circ} \mathrm{C}$ with the primary antibody against cleaved caspase-3 (1:1000; Asp 175, Cell Signaling, Danvers, MA, USA) or Bcl- $\mathrm{X}_{\mathrm{L}}$ ( $1: 1000$; Cell Signaling) prepared in 5\% non-fat dry milk solution in Tween/Tris-buffered saline. Membranes were washed with TBS (0.05\% (v/v) Tween 20 in PBS, pH 7.4) and incubated with a $1: 2000$ dilution of HRP-conjugated secondary antibody for $45 \mathrm{~min}$. Protein bands were visualized by an enhanced chemiluminescence reaction (Amersham Pharmacia Biotech, Piscataway, NJ, USA). The gray values of specific band were analyzed quantitatively using image analysis software.

\section{Immunofluorescence assay}

Paraffin sections were subjected to routine dewaxing, hydration, and antigen retrieval. Sections were incubated with primary antibody (anti-Cleaved Caspase-3 antibody, 1 :500, Asp 175; Cell Signaling) at $4{ }^{\circ} \mathrm{C}$ overnight. After $1 \times$ TBS washing, sections were further incubated with fluorescein isothiocyanate-labeled goat anti-rabbit antibody at room temperature for $45 \mathrm{~min}$. Sections were washed and incubated with PI. The reaction was terminated after rinsing, and sections were dried and mounted with $10 \%$ glycerol. All samples were observed under the laser confocal microscope (Zeiss 510).

\section{Histopathological examination and ONL thickness measurement}

Rats were killed by an overdose of intraperitoneal injection of $1 \%$ pentobarbital sodium. The cornea was labeled at the direction of 1200 hours. The bulbar conjunctiva at the limbus was retained, whereas that at the other parts was removed. The eyeballs were rapidly removed and placed in $10 \%$ neutral formalin fixative for $24 \mathrm{~h}$. Sagittal sections of optic nerve were prepared along the suture labeling (direction from 1200 to 0600). Sections were dehydrated in graded concentrations of alcohol, embedded in paraffin and cut into $5-\mu$ m-thick slices, stained with hematoxylin-eosin, and observed under a light microscope. ONL thickness (the distance from the outer plexiform layer to the outer limiting membrane) was measured using Zeiss software (Zeiss 510). Each eye was measured six times. Samples were also prepared for transmission electron microscopy: rats were killed as above and the eyeballs were rapidly removed. The vitreous cavity was removed on ice, and the retina was carefully peeled and fixed in $2 \%$ glutaraldehyde for at least $12 \mathrm{~h}$. Sections were rinsed three times with $1 \mathrm{~mol} / \mathrm{l}$ phosphate buffer solution and fixed in $1 \%$ buffered osmium tetroxide for $2 \mathrm{~h}$, followed by washing three times with distilled water, gradual dehydration in ethanol and embedding in epoxy resin. Semi-thin sections $(1 \mu \mathrm{m})$ were positioned under the light microscope and cut into ultrathin sections, followed by double staining with uranyl acetate and lead citrate. Retinal ultrastructure was observed under a transmission electron microscope (JEM-100S, Hitachi, Tokyo, Japan).

\section{Statistical analysis}

All data were expressed as means \pm SD. Analysis of variance and Dunnett's $t$-test were performed using the SAS 6.12 statistical software package (SAS Institute, Cary, NC, USA). Flash ERGs before and after injection were compared using paired $t$-test. A level of $P<0.05$ was considered statistically significant.

\section{Results}

In vivo observations of eyeball morphology and structure following intravitreal EPO injection

In vivo observations were performed under an operating microscope at 3 days after injection. All operated eye corneas were transparent, the anterior chambers showed 
no exudation, and the lenses were transparent. The vitreous body was transparent, no white dust-like crystals were observed, the ocular fundus was clearly visible, and there were no signs of retinal edema, hemorrhage, or abnormal changes in vascular morphology.

\section{Effects of intravitreal EPO injection on normal retinal function}

Flash ERGs showed no differences in latency or amplitude of flash ERG maximal response a- and b-wave before and 3 days after intravitreal injection of EPO $400 \mathrm{ng}$ (paired $t$-test, $P>0.05$ ). There were no significant differences in P4 latency or total amplitude of the sub-waves in oscillatory potentials, which reflected the function of inner retinal neurons before and after injection $(P>0.05)$.

\section{Effects of intravitreal EPO injection on normal retinal morphology and structure}

Optical microscopic examination showed normal retinal layer structure, no apparent edema or necrosis, and no change in retinal thickness at 14 days after intravitreal injection of $400 \mathrm{ng}$ EPO. The retina retained its neat arrangement, with no significant edema or necrosis and no new blood vessels within or on the surface of the retina at 2 months after injection. Transmission electron microscopy showed no significant necrosis or apoptosis throughout the whole layer of retinal neurons, the photoreceptor outer-segment disk membranes remained orderly arranged, and no apparent degeneration was seen at 3 days after injection.

\section{Influence of EPO supplementation on retinal photoreceptor apoptosis}

No yellow-stained apoptotic cells were viewed in the layers of retinal neurons in the normal control group. However, some yellow-stained apoptotic cells were seen in the ONL of the RD and RD + PBS groups. Apoptotic cells were visible, but their numbers were reduced in the RD + EPO 100, 200, and 400 ng groups (Figure 1).

\section{Influence of EPO supplementation on caspase-3 activation and $\mathrm{Bcl}-\mathrm{X}_{\mathrm{L}}$ expression after $\mathrm{RD}$}

The intensities of the gray bands representing activated caspase- 3 in the normal control, RD, RD + PBS, and $\mathrm{RD}+\mathrm{EPO} 100,200$, and $400 \mathrm{ng}$ groups at 3 days after RD were $0.16 \pm 0.04,0.55 \pm 0.14,0.54 \pm 0.11,0.35 \pm 0.08$, $0.31 \pm 0.08$, and $0.21 \pm 0.02$, respectively (Figure $2 \mathrm{a}$ ). There were significant differences among groups $(F=35.96, P<0.01)$, and the level of activated caspase-3
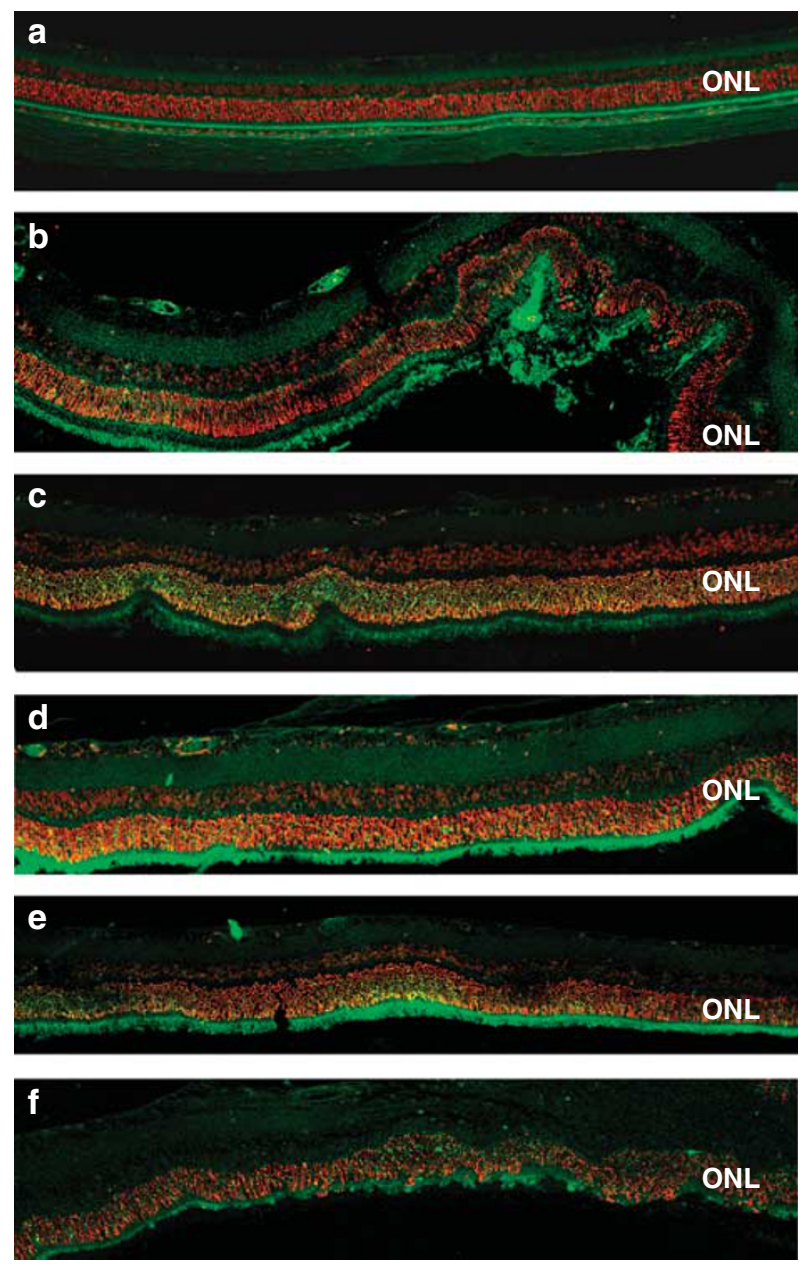

Figure 1 Photoreceptor cell apoptosis increased at 3 days after RD and was reduced after EPO treatment. (a) Normal control group; (b) RD group; (c) RD + PBS group; (d-f) RD + EPO 100, 200 , and $400 \mathrm{ng}$ groups. ONL, outer nuclear layer (TUNEL staining, $\times 200$ ).

in $\mathrm{RD}+\mathrm{EPO} 400 \mathrm{ng}$ group was significantly lower than that in other experimental RD groups $(P<0.05)$. The equivalent values for the $\mathrm{Bcl}-\mathrm{X}_{\mathrm{L}}$ bands were $0.30 \pm 0.01$, $0.24 \pm 0.03,0.27 \pm 0.05,0.45 \pm 0.02,0.43 \pm 0.04$, and $0.55 \pm 0.05$, respectively (Figure $2 b$ ). There were significant differences among groups $(F=30.75, P<0.01)$ and the expression level of Bcl- $X_{\mathrm{L}}$ in $\mathrm{RD}+\mathrm{EPO} 400 \mathrm{ng}$ group was significantly higher than that in other experimental RD groups $(P<0.05)$.

Caspase- 3 activation was also examined by tissue immunofluorescence at 3 days after intravitreal injection of EPO. There were no caspase-3-positive nuclei in normal retinas, but many yellow-stained caspase-3positive nuclei in the RD and RD + PBS groups. Reduced numbers of yellow-stained cells were seen in the RD + EPO 100, 200, and 400 ng groups (Figure 3). This was consistent with previous results of TUNEL staining. 
a

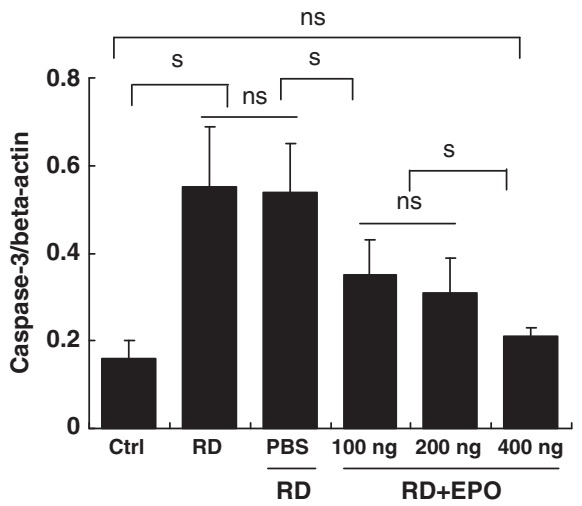

b
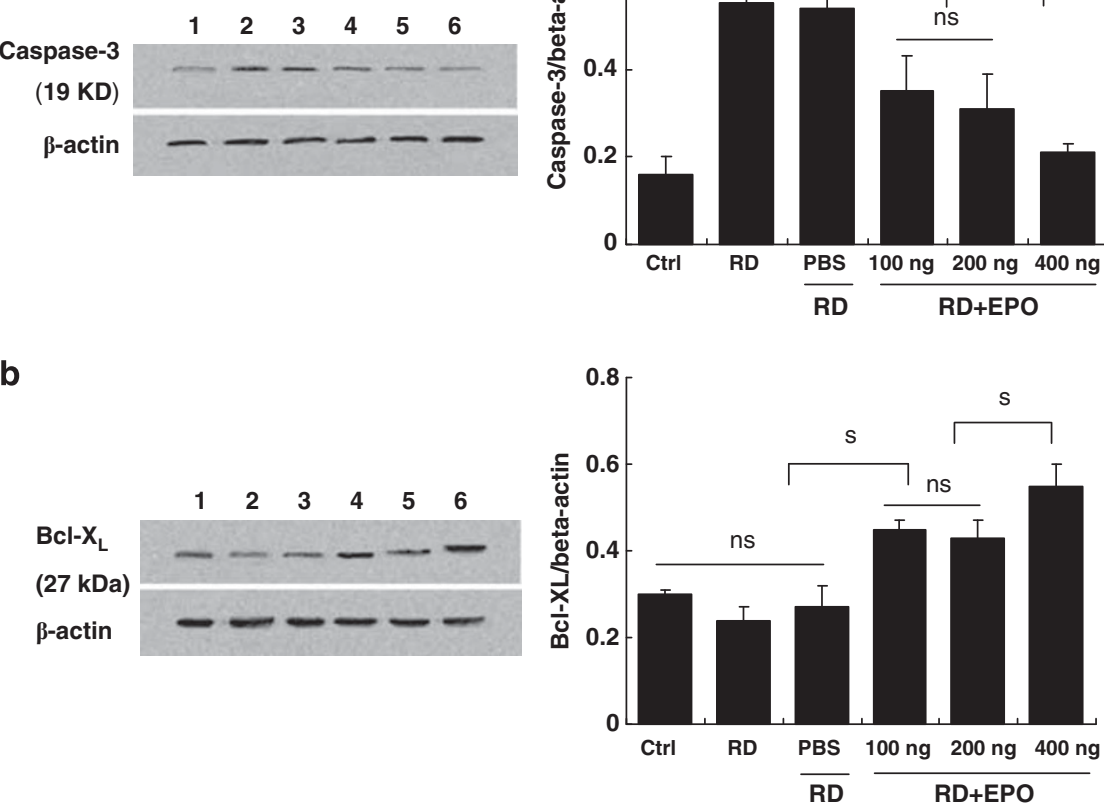

Figure 2 (a) Caspase-3 activity was significantly enhanced in the neural retina after RD and significantly reduced after EPO treatment at 3 days after RD, determined by western blotting $(n=3)$. (b) Bcl- $X_{L}$ expression was significantly enhanced after EPO treatment at 3 days after RD $(n=3)$. Lanes 1-6: normal control group, RD group, RD + PBS group, RD + EPO 100, 200, and $400 \mathrm{ng}$ groups.
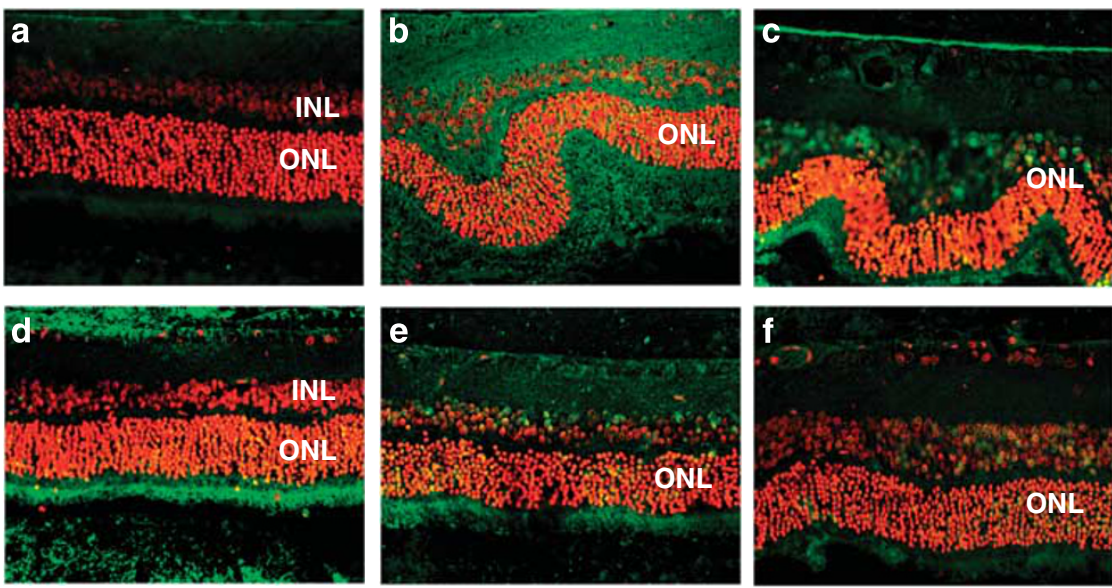

Figure 3 Caspase-3 activity was significantly enhanced in the neural retina at 3 days after RD and significantly reduced after EPO treatment $(n=3)$. (a) Normal control group; (b) RD group; (c) RD + PBS group; (d-f) RD + EPO 100, 200, and 400 ng groups. INL, inner nuclear layer; ONL, outer nuclear layer (immunofluorescence, $\times 400$ ).

\section{Effects of EPO supplementation on structure and morphology of the detached retina and ONL thickness}

No significant histopathological changes were detected in the inner retina at 14 days after RD, but obvious changes were detected in the outer neural retina. Both inner and outer segments of photoreceptor cells were disordered and shortened. The ONL thickness was slightly reduced following RD, but was thicker in the EPO treatment groups (Figure 4). ONL thickness was measured using image analysis software and the thicknesses in the normal control, RD, RD + PBS, and 

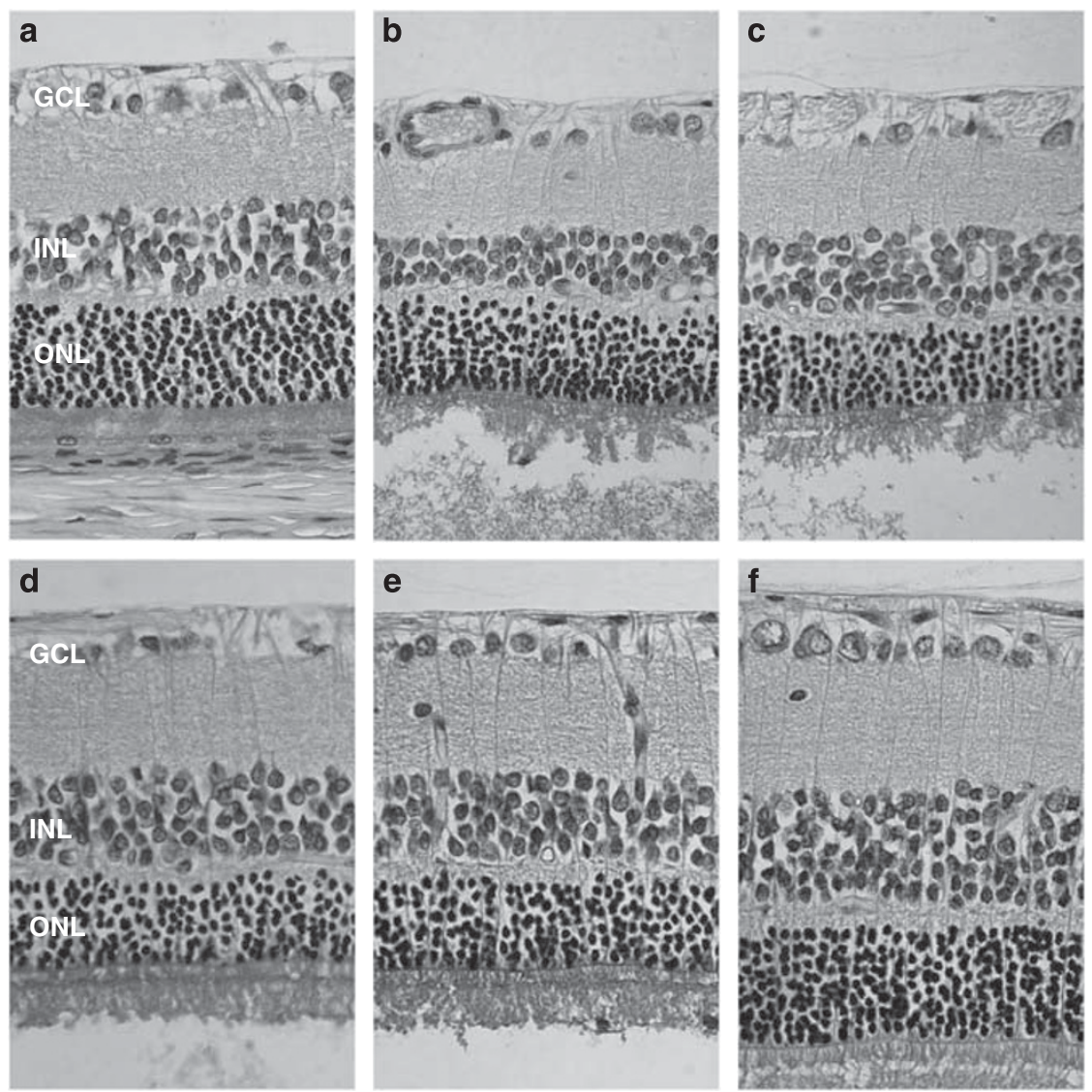

Figure 4 At 14 days after RD, the thickness of the ONL was reduced and the photoreceptor outer segment collapsed and disappeared. The ONL in the EPO groups was thicker than that in the RD and RD+ PBS groups, examined under an optical microscope $(n=6)$. (a) Normal control group; (b) RD group; (c) RD + PBS group; (d-f) RD + EPO 100, 200, and 400 ng groups. GCL, ganglion cell layer; INL, inner nuclear layer; ONL, outer nuclear layer (hematoxylin-eosin staining, $\times 400$ ).

$\mathrm{RD}+\mathrm{EPO} 100,200$, and $400 \mathrm{ng}$ groups were $45.70 \pm 1.53$, $35.00 \pm 1.66,34.79 \pm 1.15,34.60 \pm 1.02,36.82 \pm 1.23$, and $40.20 \pm 2.41 \mu \mathrm{m}$, respectively. Analysis of variance showed significant differences among groups $(F=21.52$, $P<0.01)$. The ONL thickness in the RD + EPO $400 \mathrm{ng}$ group was statistically significant compared with that in other experimental RD groups $(P<0.05)$. At 2 months after RD, there were still no apparent histopathological changes in the inner retina, but the ONL thickness was greatly reduced (Figure 5). The ONL thicknesses in each group were $46.74 \pm 1.97,14.51 \pm 1.42,14.70 \pm 1.19$, $18.17 \pm 1.23,26.28 \pm 4.25$, and $34.00 \pm 2.40 \mu \mathrm{m}$,

respectively. Analysis of variance demonstrated statistically significant differences among groups $(F=96.25, P<0.01)$. The ONL thickness in the RD + EPO $400 \mathrm{ng}$ group was statistically significant compared with that in other experimental groups $(P<0.05)$. Interestingly, the lengths and alignments of the inner and outer sections of photoreceptor cells were better in the EPO treatment groups than in the $\mathrm{RD}$ and $\mathrm{RD}+\mathrm{PBS}$ groups.

\section{Discussion}

Hypoxic preconditioning, transgenic animals expressing endogenous EPO, and EPO supplementation have demonstrated strong neuroprotective effects in many models of optic nerve retinal diseases. However, the effects of increased EPO levels on normal tissue metabolism, structure, and function remain unclear. It is therefore essential to identify the effects of intravitreal $E P O$ injection on changes in retinal structure and function. Flash ERG demonstrated that intravitreal injection of $400 \mathrm{ng}$ EPO had no significant effects on the amplitude or latency of the maximum response wave or oscillatory potentials, indicating a lack of effect on retinal function. Histopathological examination also showed no effect of EPO on retinal layer structure, retinal thickness, retinal edema or necrosis, or retinal neovascularization at 14 days and 2 months after injection. These results indicate that intravitreal injection of up to $400 \mathrm{ng}$ of EPO does not affect the structure or function of the retina. 

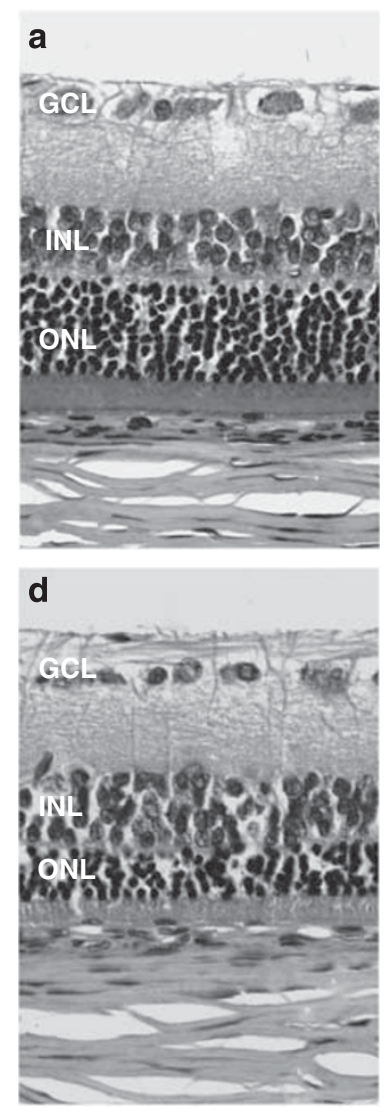
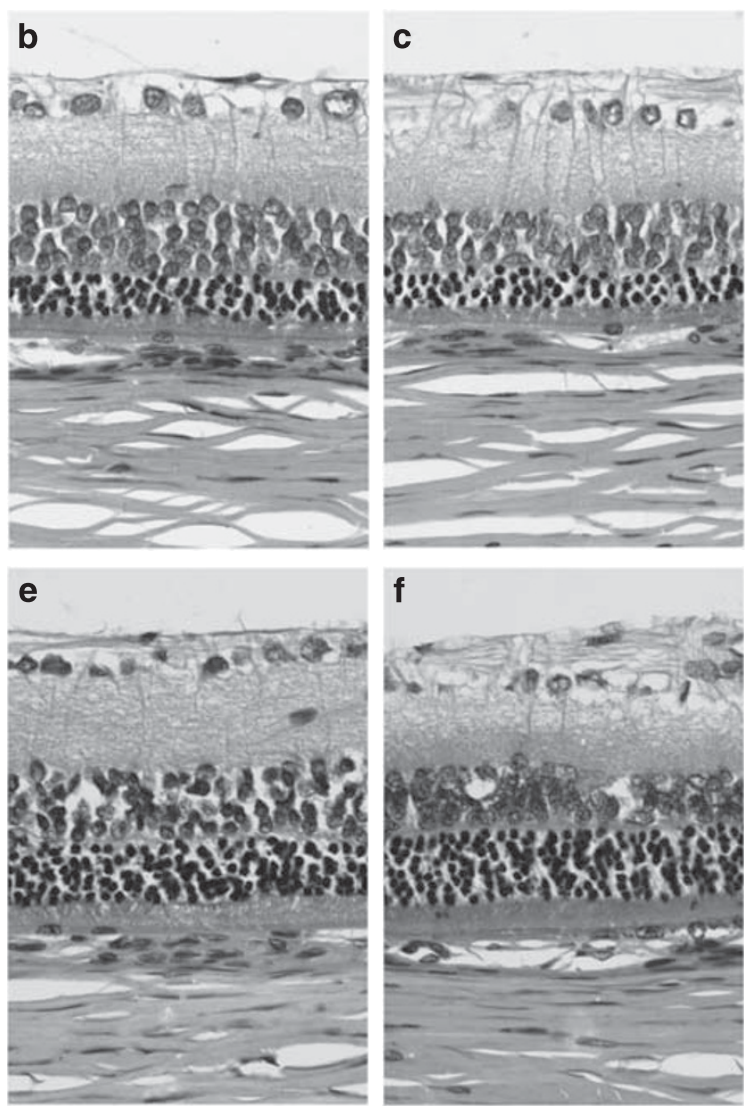

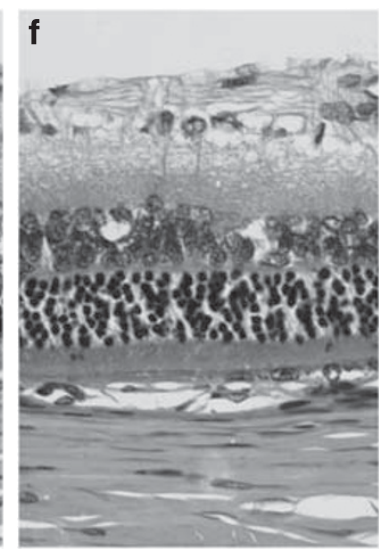

Figure 5 All retinas were reattached at 2 months after RD, as shown by optical microscopy. The thickness of the ONL was reduced, but the ONL was still thicker in the EPO treatment groups compared with the RD and RD + PBS groups. The effect of EPO was dose-dependent $(n=6)$. (a) Normal control group; (b) RD group; (c) RD + PBS group; (d-f) RD + EPO 100, 200, and 400 ng groups. GCL, ganglion cell layer; INL, inner nuclear layer; ONL, outer nuclear layer (hematoxylin-eosin staining, $\times 400$ ).

These preliminary results confirmed the hypothesis of Tsai $e t a l,{ }^{8}$ who reported that intravitreal injection of up to $625 \mathrm{ng}$ of EPO had no effect on retinal function in rats. The effect of EPO on angiogenesis is another potential issue of concern. Takagi et $a l^{30}$ demonstrated that EPO was a major angiogenic factor in proliferative diabetic retinopathy, independent of VEGF. Chen $e t a^{20}$ showed that decreased EPO production in early hypoxic retinopathy could cause retinal vascular instability, and that elevated levels of EPO during the proliferative period could lead to retinal neovascularization. No retinal neovascularization was detected by light microscopy at 2 months after EPO injection, but the impact of EPO on retinal capillaries requires further investigation.

Apoptosis is the main mechanism of photoreceptor cell death after RD, and both the mitochondrial and Fas/FasL pathways are involved in the apoptotic process. ${ }^{31,32}$ This study showed that exogenous EPO after RD significantly reduced the number of TUNEL-positive cells, indicating that EPO partially inhibited apoptosis. Caspase- 3 is the final executor of apoptosis and is also involved in photoreceptor cell apoptosis after RD. Western blot in the present study showed that activated caspase-3 expression was significantly increased after RD, in accordance with previous studies (data unpublished). The results also revealed that exogenous EPO significantly upregulated the expression of Bcl- $\mathrm{X}_{\mathrm{L}}$ and reduced the expression of caspase-3, indicating that the combination of EPO and EPOR may induce Bcl-2 antiapoptotic gene expression by signal transduction pathways, and inhibits apoptosis by suppressing the mitochondrial release of cytochrome $\mathrm{C}$ and regulating intracellular $\mathrm{Ca}^{2+}$ levels. Histopathological findings revealed that the ONL thickness in all groups was only slightly reduced at $14 \mathrm{~d}$ after $\mathrm{RD}$; although this reduction was significant, it was less than that reported in previous studies, such as those of Berglin et $a l^{33}$ and Yang et al, ${ }^{34}$ who reported that the ONL thickness was reduced by half at 14 days after local RD in rabbits. This variation may be due to inter-species differences, the concentrations of sodium hyaluronate used, or the retinal 
uplift height. The majority of retinas were reattached at 28 days after $\mathrm{RD}$, and all detached retinas were reattached at 2 months. Regarding the morphology, ONL thickness was significantly thicker in the EPO treatment groups, compared with the control group. In addition, the length and arrangement of the inner and outer segments of photoreceptor cells after EPO treatment were less disrupted than in the RD and RD + PBS groups at 14 days and 2 months after RD, suggesting that EPO has a role in maintaining the morphologies of the inner and outer segments of photoreceptor cells, though the underlying mechanism remains unclear.

In conclusion, this preliminary study investigated the safety, efficacy, and underlying mechanism of exogenous EPO in terms of its protective effect on photoreceptor cells after RD. The results demonstrated that intravitreal injection of EPO $400 \mathrm{ng}$ had no apparent effects on retinal morphology or function. In addition, EPO suppressed caspase- 3 activation and increased $\mathrm{Bcl}-\mathrm{X}_{\mathrm{L}}$ expression, thereby protecting the retinal photoreceptor cells from RD-induced apoptosis. The results suggest that clinical supplementation with exogenous EPO may exert some protective effect against ischemic retinal diseases, such as diabetic retinopathy, glaucoma, and retinal vein occlusion.

\section{Summary}

\section{What was known before}

- Erythropoietin (EPO) has been shown to protect the retinal neurons in retinal diseases.

\section{What this study adds}

- Intravitreal injection of EPO $400 \mathrm{ng}$ is safe in terms of its effects on retinal morphology and function in normal rats.

- Intravitreal EPO may suppress caspase-3 activation and enhance $\mathrm{Bcl}-\mathrm{X}_{\mathrm{L}}$ expression, resulting in inhibition of apoptosis and protection of photoreceptor cells.

\section{Conflict of interest}

The authors declare no conflict of interest.

\section{Acknowledgements}

This study was supported by a grant from the Shanghai Key Laboratory of Ocular Fundus Disease.

\section{References}

1 Jelkmann W, Metzen E. Erythropoietin in the control of red cell production. Ann Anat 1996; 178: 391-403.

2 Rex TS, Allocca M, Domenic L, Surace EM, Maguire AM, Lyubarsky A et al. Systemic but not intraocular EPO gene transfer protects the retina from light- and genetic-induced degeneration. Mol Thr 2004; 10: 855-861.
3 Grimm C, Wenzel A, Stanescu D, Samardzija M, Hotop S, Groszer $\mathrm{M}$ et al. Constitutive overexpression of human erythropoietin protects the mouse retina against induced but not inherited retinal degeneration. J Neurosci 2004; 24: 5651-5658.

4 Girmm C, Wenzel A, Groazer M, Mayser H, Seeliger M, Samardzija M et al. HIF-1-induced erythropoietin in the hypoxic retina protects against light-induced retinal degeneration. Nat Med 2002; 8: 718-724.

5 Rex TS, Wong Y, Kodali K, Merry S. Neuroprotection of photoreceptors by direct delivery of erythropoietin to the retina of the retinal degeneration slow mouse. Exp Eye Res 2009; 89: 735-740.

6 Junk AK, Mammis A, Savitz SI, Singh M, Roth S, Malhotra S et al. Erythropoietin administration protects retinal neurons from acute ischemia-reperfusion injury. Proc Natl Acad Sci USA 2002; 99: 10659-10664.

7 Fu QL, Wu W, Wang H, Li X, Lee VW, So KF. Up-regulated endogenous erythropoietin /erythropoietin receptor system and exogenous erythropoietin rescue retinal ganglion cells after chronic ocular hypertension. Cell Mol Neurobiol 2008; 28: 317-329.

8 Tsai JC, Wu L, Worgul B, Forbes M, Cao J. Intravitreal administration of erythropoietin and preservation of retinal ganglion cells in an experimental rat model of glaucoma. Curr Eye Res 2005; 30: 531-538.

9 Tsai JC, Song BJ, Wu L, Forbes M. Erythropoietin: A candidate neuroprotective agent in the treatment of glaucoma. J Glaucoma 2007; 16: 567-571.

10 Weishaupt JH, Rohde G, Pölking E, Siren AL, Ehrenreich H, Bähr M. Effect of erythropoietin axotomy-induced apoptosis in rat retinal ganglion cells. Invest Ophthalmol Vis Sci 2004; 45: 1514-1522.

11 Kilic Ü, Kilic E, Soliz J, Bassetti CI, Gassmann M, Hermann DM. Erythropoietin protects from axotomyinduced degeneration of retinal ganglion cells by activating ERK-1/-2. FASEB J 2005; 19: 249-251.

12 King CE, Rodger J, Bartlett C, Esmaili T, Dunlop SA, Beazley LD. Erythropoietin is both neuroprotective and neuroregenerative following optic nerve transaction. Exp Eye Res 2007; 205: 48-55.

13 Sättler MB, Merkler D, Maier K, Stadelmann C, Ehrenreich H, Bähr M et al. Neuroprotective effects and intracellular signaling pathways of erythropoietin in a rat model of multiple sclerosis. Cell Death Differ 2004; 11: 181-192.

14 Diem R, Sättler MB, Merkler D, Demmer I, Maier K, Stadelmann $\mathrm{C}$ et al. Combined therapy with methylprednisolone and erythropoietin in a model of multiple sclerosis. Brain 2005; 128: 375-385.

15 Zhu B, Wang W, Gu Q, Xu X. Erythropoietin protects retinal neurons and glial cells in early-stage streptozotocin-induced diabetic rats. Exp Eye Res 2008; 86: 375-382.

16 Zhang JF, Wu Y, Jin Y, Ji F, Sinclair SH, Luo Y et al. Intravitreal injection of erythropoietin protects both retinal vascular and neuronal cells in early diabetes. Invest Ophthalmol Vis Sci 2008; 9: 732-742.

17 Shen J, Wu Y, Xu JY, Zhang J, Sinclair SH, Yanoff M et al. ERK- and Akt-dependent neuroprotection by erythropoietin (EPO) against glyoxal-AGEs via modulation of Bcl-xL, Bax, and BAD. Invest Ophthalmol Vis Sci 2010; 51: 35-46.

18 Wang Q, Pfister F, Dorn-Beineke A, vom Hagen F, Lin J, Feng $\mathrm{Y}$ et al. Low-dose erythropoietin inhibits oxidative 
stress and early vascular changes in the experimental diabetic retina. Diabetologia 2010; 53: 1227-1238.

19 Li W, Sinclair SH, Xu GT. Effects of intravitreal erythropoietin therapy for patients with chronic and progressive diabetic macular edema. Ophthalmic Surg Lasers Imaging 2010; 41: 18-25.

20 Chen J, Connor KM, Aderman CM, Smith LE. Erythropoietin deficiency decreases vascular stability in mice. J Clin Invest 2008; 118: 526-533.

21 Patel S, Rowe MJ, Winters SA, Ohls RK. Elevated erythropoietin mRNA and protein concentrations in the developing human eye. Pediatr Res 2008; 63: 394-397.

22 Chung H, Lee H, Lamoke F, Hrushesky WJ, Wood PA, Jahng WJ. Neuroprotective role of erythropoietin by antiapoptosis in the retina. J Neurosci Res 2009; 87: 2365-2374.

23 Juul SE, Yachnis AT, Christensen RD. Tissue distribution of erythropoietin and erythropoietin receptor in the developing human fetus. Early Hum Dev 1998; 52: 235-249.

24 García-Ramírez M, Hernández C, Simó R. Expression of erythropoietin and its receptor in the human retina: a comparative study of diabetic and nondiabetic subjects. Diabetes Care 2008; 31: 1189-1194.

25 Shah SS, Tsang SH, Mahajan VB. Erythropoetin receptor expression in the human diabetic retina. BMC Res Notes 2009; 2: 234

26 Anderson DH, Guérin CJ, Erickson PA, Stern WH, Fisher SK. Morphological recovery in the reattached retina. Invest Ophthalmol Vis Sci 1986; 27: 168-172.

27 Xie Z, Wu X, Qiu Q, Gong Y, Song Y, Gu Q et al. Expression pattern of erythropoietin and erythropoietin receptor in experimental model of retinal detachment. Curr Eye Res 2007; 32: 757-764.

28 Wang ZY, Shen LJ, Zhao KK, Song ZM, Qu J. Elevated erythropoietin in vitreous of patients with rhegmatogenous retinal detachment and proliferative vitreoretinopathy. Ophthalmic Res 2009; 42: 138-140.

29 Xie Z, Wu X, Gong Y, Song Y, Qiu Q, Li C. Intraperitoneal injection of Ginkgo Biloba extract (EGb 761) enhances anti-oxidation capability of retina and protects photoreceptors after light-induced retinal damage in rats. Curr Eye Res 2007; 32: 471-479.

30 Takagi H, Watanabe D, Suzuma K, Kurimoto M, Suzuma I, Ohashi $\mathrm{H}$ et al. Novel role of erythropoietin in proliferative diabetic retinopathy. Diabetes Res Clin Pract 2007; 77: S62-S64.

31 Zacks DN, Hänninen V, Pantcheva M, Ezra E, Grosskreutz C, Miller JW. Caspase activiation in an experimental model of retinal detachment. Invest Ophthalmol Vis Sci 2003; 44: 1262-1267.

32 Zacks DN, Zheng QD, Han Y, Bakhru R, Miller JW. FAS-mediated apoptosis and its relation to intrinsic pathway activation in an experimental model of retinal detachment. Invest Ophthalmol Vis Sci 2004; 45: 4563-4569.

33 Berglin L, Algvere PV, Seregard S. Photoreceptor decay over time and apoptosis in experimental retinal detachment. Graefe's Arch Clin Exp Ophthalmol 1997; 235: 306-312.

34 Yang L, Bula D, Arroyo JG, Chen DF. Preventing retinal detachment-associated photoreceptor cell loss in Bax-deficient mice. Invest Ophthalmol Vis Sci 2004; 45: 648-654. 